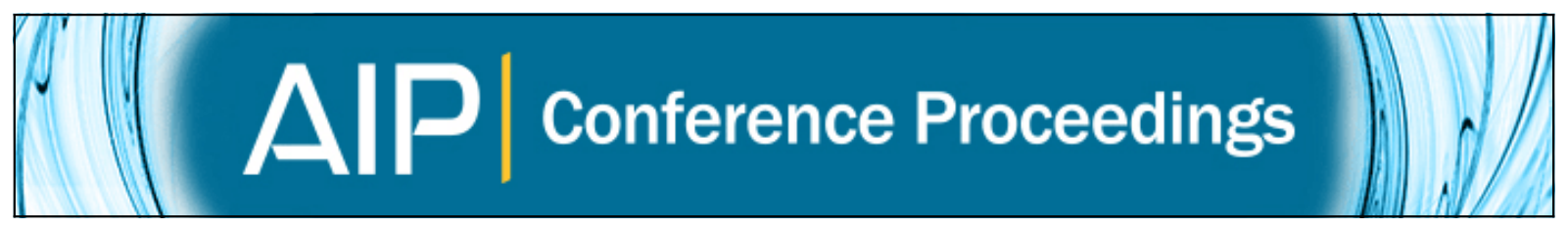

\title{
Properties of Plasma Jets Emitted in Pulsed Capillary Discharges at Low Pressures
}

G. Avaria, H. Bhuyan, M. Favre, H. Chuaqui, I. Mitchell, E. Wyndham, and D. Grondona

Citation: AIP Conference Proceedings 875, 393 (2006); doi: 10.1063/1.2405972

View online: http://dx.doi.org/10.1063/1.2405972

View Table of Contents: http://scitation.aip.org/content/aip/proceeding/aipcp/875?ver=pdfcov

Published by the AIP Publishing

Articles you may be interested in

Observation of plasma jets in a table top plasma focus discharge

Phys. Plasmas 22, 040705 (2015); 10.1063/1.4919260

Electron dynamics and plasma jet formation in a helium atmospheric pressure dielectric barrier discharge jet Appl. Phys. Lett. 99, 121501 (2011); 10.1063/1.3628455

Optical and electrical characterization of an atmospheric pressure microplasma jet with a capillary electrode Phys. Plasmas 17, 033502 (2010); 10.1063/1.3330507

A simple cold Ar plasma jet generated with a floating electrode at atmospheric pressure

Appl. Phys. Lett. 93, 011503 (2008); 10.1063/1.2956411

Properties of Plasma Jets Emitted in Pulsed Capillary Discharges

AIP Conf. Proc. 808, 247 (2006); 10.1063/1.2159363 


\title{
Properties of Plasma Jets Emitted in Pulsed Capillary Discharges at Low Pressures
}

\author{
G. Avaria*, H. Bhuyan*, M. Favre', H. Chuaqui*, I. Mitchell*, E. Wyndham* and D. \\ Grondona $^{2 \dagger}$
}

*Departamento de Física, Pontificia Universidad Católica de Chile, Casilla 306, Santiago 22, Chile

${ }^{\dagger}$ Departamento de Fisica, Facultad de Ciencias Exactas y Naturales, Instituto de Física del Plasma (CONICET), Universidad de Buenos Aires, Ciudad Universitaria Pabellón 1, 1428, Buenos Aires, Argentina

\begin{abstract}
The characteristic geometry of a pulsed capillary discharge (PCD), a narrow open end tube with a large aspect ratio, establishes natural conditions for the generation of plasma jets, that propagate in the neutral background gas surrounding the capillary. We have investigated the plasma jet emission in a PCD, with electron beam assisted on-axis initiation. A local storage capacitor, coaxial with the capillary assembly, is pulse charged up to a maximum of $-11 \mathrm{kV}$, which results in an initial $\sim 10 \mathrm{~ns}, \sim 2 \mathrm{kA}$ current pulse. The discharge is operated in Argon and Nitrogen, in a continuous pulsing mode, at frequencies between 5 to $20 \mathrm{~Hz}$, and in a pressure range between 300 to 1100 Torr. A fast Langmuir probe, placed at the anode side, close to the capillary exit, is used to measure the characteristic electron temperature $\left(T_{e}\right)$ and electron density $\left(N_{e}\right)$ of the plasma jets. Results obtained in Nitrogen at $10 \mathrm{~Hz}$ indicate that $T_{e}$ is in the 10 to $60 \mathrm{eV}$ range with $N_{e}$ of the order of $10^{14}\left(\mathrm{~cm}^{-3}\right)$. Significant changes in the probe floating potential are observed for a fixed operating pressure when the pulsing frequency is varied, which can be ascribed to the presence of metastables in the gas fed into the capillary, which result from the interaction of the plasma jet with the plenum gas, at the cathode side. The presence of metastables reduces the energy cost involved in the capillary plasma production, which results in a pulsing frequency dependence of the plasma parameters.
\end{abstract}

Keywords: capillary discharge, plasma jets.

PACS: $52.25 .-\mathrm{b}, 52.50 . \mathrm{Dg}, 52.80 . \mathrm{Tn}$

\section{INTRODUCTION}

Pulsed capillary discharge (PCD) devices are transient plasma sources, which have been initially investigated as pulsed radiation sources emitting in the extreme ultraviolet to soft $x$-ray region (XUV) [1]. Further work in PCD has also been reported in the context of laser gain in the vacuum ultraviolet (VUV), at $46.9 \mathrm{~nm}$ [2], laser pulse transport through plasma guides [3] and XUV radiation sources for lithography [4]. PCD operating in gas media require some kind of discharge initiation, which favors on-axis plasma formation to avoid plasma contamination by wall ablated material. We have previously reported on a PCD with electron beam assisted on-axis initiation in which axial electron beams are produced naturally due to the hollow cathode effect [5]. Transient plasma formation inside the capillary results in the emission of high density plasma jets emitted from the open ends of the capillary, that propagate in the external neutral background gas. Time and space evolution of plasma parameters in the plasma jets at high pressures have been studied by Caballero et al. [7]. To evaluate potential applications of PCDs at a high repetitive scheme at low pressures, the

1 mfavre@fis.puc.cl

${ }^{2}$ Member of CONICET plasma parameters have been measured with a fast Langmuir probe. The experiments have been conducted in Argon and Nitrogen, in a pressure range between 0.3 to 1.1 mTor, with pulsing frequencies between 5 to $20 \mathrm{~Hz}$.

\section{EXPERIMENTAL DETAILS}

The experiments were performed in a PCD with a low inductance high voltage discharge geometry. Most of the capillary length is surrounded by a tight conducting shield, which is kept at ground potential and provides the current return path. The capillary used in these investigations is an alumina tube, $55 \mathrm{~mm}$ long, $1.6 \mathrm{~mm}$ internal diameter, located on axis between the two electrodes. The local storage capacity is $\sim 1 \mathrm{nF}$. Further details about the experimental set-up and available diagnostics can be found elsewhere [5]. The local storage capacitor is pulse charged up to $\sim 11 \mathrm{kV}$, using a novel IGBT based charger [6]. Discharges in Argon and Nitrogen were made, injecting the gas continuously at the high voltage side of the capillary, which corresponds to the cathode electrode, and pumped from the anode side, thus keeping a pressure gradient along the capillary tube. In these experiments the pressure in the hollow cathode region (HCR), the cathode side, was varied between 0.3 and 1.1 Torr, meanwhile the pressure at the anode side was 
kept between 10 and $40 \mathrm{mTorr}$, by adjusting the pumping rate. This pressure gradient is used to enhance the electron beam emission in the pre-breakdown phase of the discharge, due to the formation of a pre-breakdown hollow cathode plasma [5].

To investigate the plasma properties of the capillary plasma jets at low pressures, a Langmuir probe diagnostic was used. The discharge was operated in a repetitive mode, using two schemes:

1. Fixed Pressure $\rightarrow$ Varying Frequency

2. Fixed Frequency $\rightarrow$ Varying Pressure

The probe is a Tungsten wire, $5.5 \mathrm{~mm}$ long, $100 \mu \mathrm{m}$ diameter, placed $5 \mathrm{~mm}$ away from the capillary end. $\mathrm{I}-\mathrm{V}$ characteristics of the plasma for the schemes used were obtained by averaging the probe current signal over three series of sixty four shots, for each probe voltage. Electron temperature and plasma density were inferred from the I-V characteristic. Emission spectra were taken with an Ocean Optics PC2000 spectrometer, with a 600 $1 / \mathrm{mm}$ gratting and a $300-1000 \mathrm{~nm}$ spectral range.

\section{RESULTS AND DISCUSSION}

In figure 2, five probe signals are superimposed, corresponding to different bias voltages. The first negative peak is associated with the pre-breakdown electron beam generated in hollow cathode assisted capillary discharges [5]. It can be seen that changes in the bias voltage does not affect the rising edge of the electron beam signal, and only minor changes are seen in the peak value. Nevertheless, signal changes can be seen at the second and third peak, which respond to the variation of biasing voltage. The third peak is very sensitive to this variations, were the signal changes polarity according

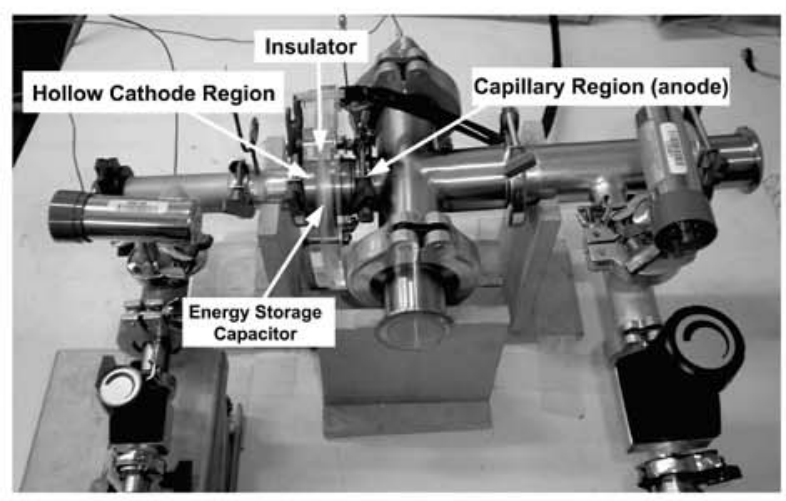

FIGURE 1. Experimental Setup to the bias voltage. We ascribe this part of the probe signal to the plasma flow associated with the capillary jet. From this signal peak, I-V characteristics can be obtained (figures 3 and 4). Each point is generated from the average of 64 shots over 3 different series. Ion and electron saturation can be seen clearly, as well as the plasma and floating potentials, $V_{p}$ and $V_{f}$, respectively. From this figures it can be seen that the latter is sensible to changes, both in frequency and pressure.

The behavior of electron temperature and plasma density was obtained for Argon and Nitrogen discharges. Figures 5 and 6 shows the variation of this features over frequency and pressure changes. In Argon, an increase

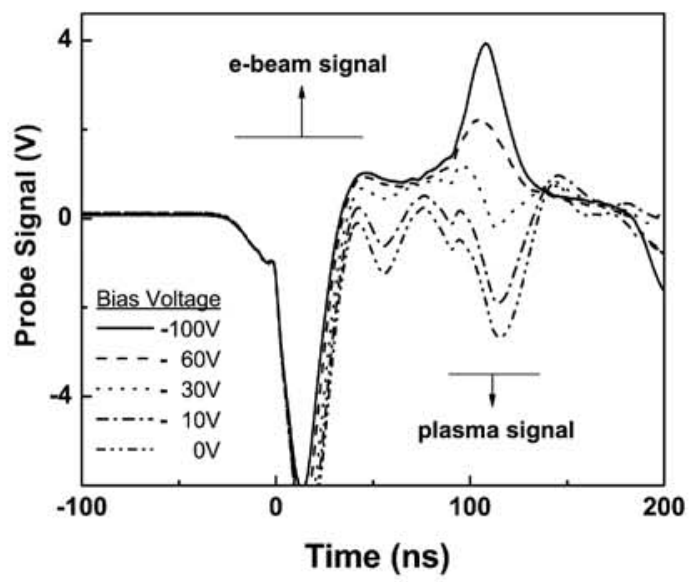

FIGURE 2. Langmuir probe signal for Nitrogen

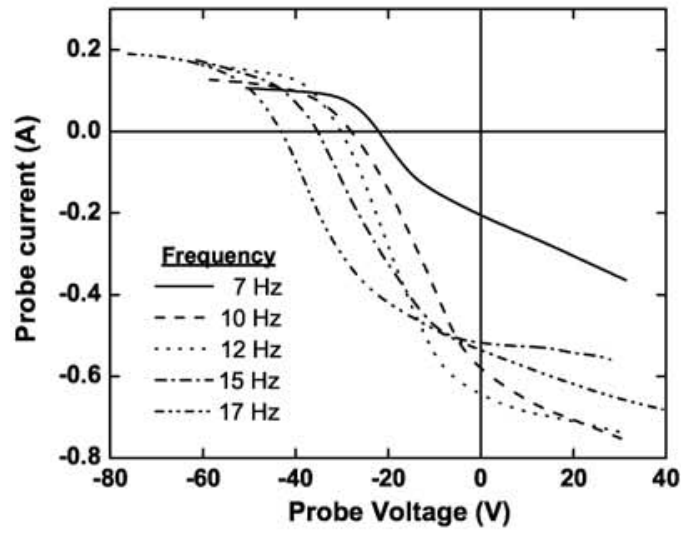

FIGURE 3. I-V characteristics for Argon, at the "varying frequency" scheme. 
in density with increasing frequency is observed. On the contrary, the electron temperature shows a small decreasing tendency. This observations are rather preliminary, due to the small range of frequencies investigated. In the "varying pressure" scheme for Argon, decreasing values for density were obtained for the pressure range investigated. Preliminary results indicate that the temperature decreases as the pressure increases.

Nitrogen discharges in the "varying pressure" scheme shows a decrease in density and temperature with increasing pressure, as it is also seen in Argon discharges. Pressures higher than 0.7 Torr could not be used for the present probe settings, due to changes in the jet evolution, caused by the fact that the Langmuir probe acts as a subsidiary anode for the discharge. Results with varying

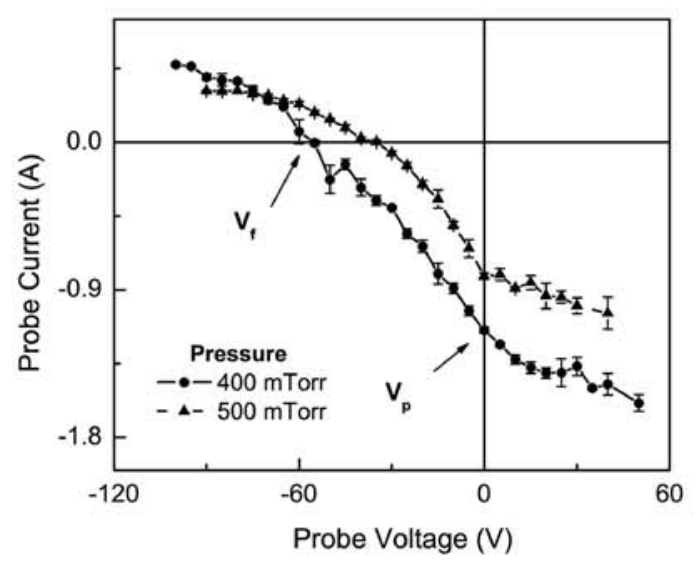

FIGURE 4. I-V characteristics for Nitrogen, at the "varying pressure" scheme.

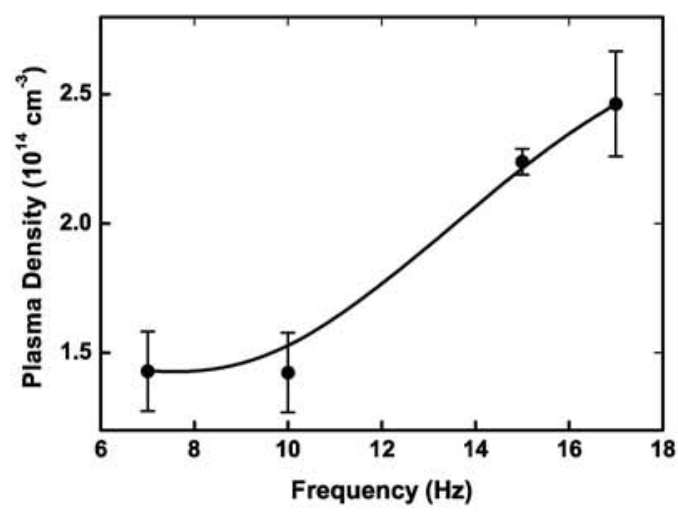

FIGURE 5. Plasma density for Argon at $700 \mathrm{~m}$ Torr frequency shows a slight increase in density, similar to the increase observed in Argon. The temperature variation tendency with frequency is also not clear, but the characteristic values are in the range obtained in the "varying pressure" scheme.

The floating potential variation with frequency could be associated with the presence of Argon metastables states in the hollow cathode region of the chamber, from where the working gas is injected. In fact, a plasma jet is also ejected from the cathode side, due to the presence of a hollow cathode. This plasma jet interacts with the high pressure neutral gas, which is being fed into the capillary volume. In the case of Argon there are two important metastables states, the $1 s_{3}$ and $1 s_{5}$ (Paschen notation), that have lifetimes of the order of seconds, $>1.3 \mathrm{~s}$ and $38 \mathrm{~s}$ respectively $[8,9,10]$. Depending on the high voltage pulsing frequency, a different percentage of Argon atoms in the first exited level is expected to be present in the capillary gas when the high voltage pulse is applied. The presence of metastables reduces the amount of energy required for ionization by electron impact and increases the characteristic cross section for the process, which might lead to a higher electron density of the capillary plasma at electric breakdown. As the floating potential is a measure of the potential when the probe draws no current from the plasma, the decrease of that potential(from $\sim 15$ to $\sim 40 \mathrm{~V}$ approx.), when the pulsing frequency increases, might be due to an increase in the electron density of the capillary jet, as compared with identical pressure condition at lower frequency. This effect is at present being studied in detail, extending the pulsing frequency up to hundreds of Hertz.

An emission spectra was taken from the jet plasma,

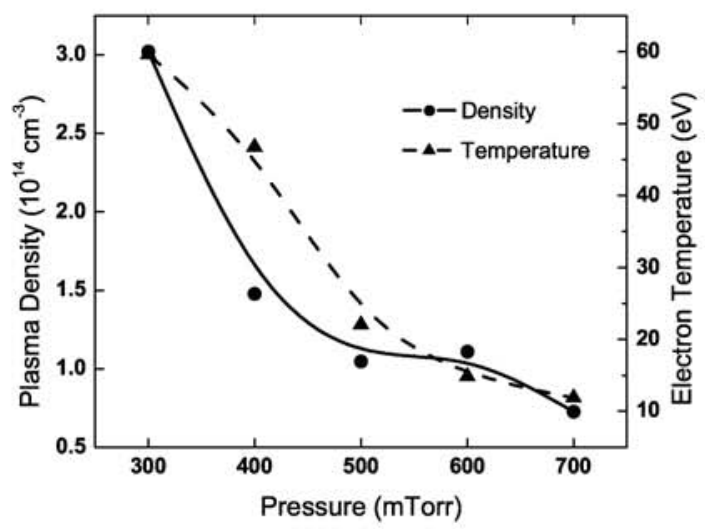

FIGURE 6. Plasma density and electron temperature for nitrogen at $10 \mathrm{~Hz}$ 
at 0.4 Torr of Nitrogen, $10 \mathrm{~Hz}$ repetition rate. This spectrum (figure 7) shows the presence of atomic copper and molecular nitrogen lines. Copper spectral lines could be associated with micro arcs formed at the rim of the brass made anode return shield. This metallic plasma contributes to the jet plasma content. The evaporated anode material, mainly copper, might deposit onto the Langmuir probe, but it does not change its measuring properties, as shown by Weaver et al. [11]. Molecular lines of nitrogen could be explained by the long integration time of the spectrometer, set to 15 seconds. Due to this, the spectrum represents the emission history over many complete cycles of the discharge, thus including emission from the capillary jets and from the recombining anode plasma, left behind after the capillary jets are extinguished.

\section{CONCLUSIONS}

Our preliminary results show that PCD is a convenient plasma source, which produces plasma jets with a wide range of characteristic plasma parameters, at a very low cost of stored energy. Our Langmuir probe measurements show an electron temperature in the range of 10 to $60 \mathrm{eV}$ with densities in the order of $10^{14} \mathrm{~cm}^{-3}$ for Nitrogen and Argon, in the pressures and frequencies ranges investigated. Further work is underway to further characterize the PCD plasma jets, and to valuate their potential in the context of surface science applications.

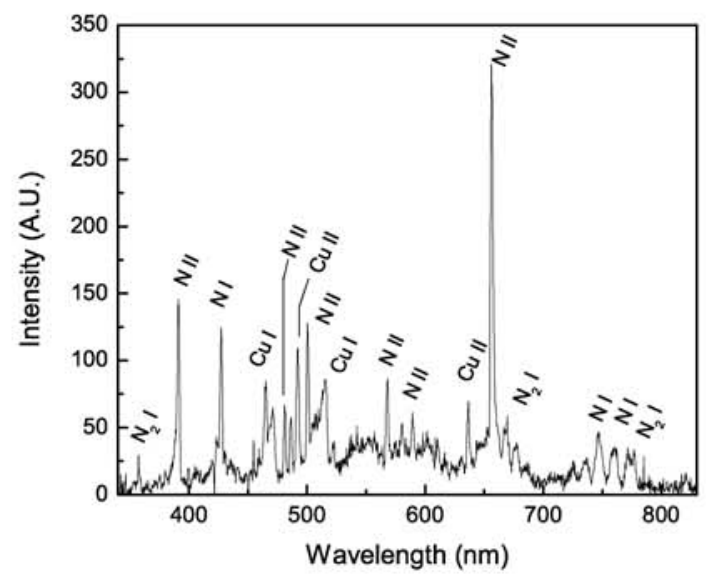

FIGURE 7. Nitrogen plasma spectrum at $10 \mathrm{~Hz}, 400 \mathrm{mTorr}$

\section{ACKNOWLEDGMENTS}

This work has been founded by FONDECYT grants $\# 1030970$ and \#7050131. G. Avaria is supported by a doctoral fellowship from project MECESUP \#00006, and wishes to thank N. Rodriguez for all the help given.

\section{REFERENCES}

1. P. Bogen, H. Conrads, G. Gatti, and W. Kohlhass, J. Opt. Soc. Am. A 58, 203 (1968)

2. J. J. Rocca, V. Shlyaptsev, F. G. Tomasel, O. D. Cortázar, D. Hartshorn, and J. L. A: Chilla, Phys. Rev. Lett. 73, 2192 (1994)

3. D. Kaganovish, P.V. Sasorov, C. Cohen and A. Zigler, Appl. Phys. Lett. 75, 772 (1999)

4. I. Krisch, P. Choi, J. Larour, M. Favre, J. Rous and C. Leblanc, Contrib. Plasma Phys. 40, 135 (2000)

5. P. Choi and M. Favre, Rev. Sci. Instrum. 69, 3118 (1998)

6. P. Choi, E. Wyndham and Y. An, IEEE Int. Conf. on Pulse Power, Monterrey, CA, U.S.A., 14-17 June, 2005, paper \#10059

7. L.S. Caballero, H. Chuaqui, M. Favre, I. Mitchell, and E. Wyndham, J. Appl. Phys. 98, 023305 (2005)

8. J. B. Boffard, G. A. Piech, M. F. Gehrke, L. W. Anderson, and C. C. Lin, Phys. Rev. A 59, 2749 (1999).

9. J. R. S. Van Dyck, C. E. Johnson, and H. A. Shugart, Phys. Rev. $A$ 5, 991 (1972)

10. H. Katori, and F. Shimizu, Phys. Rev. Lett. 70, 3545 (1993)

11. I. Weaver, G. W. Martin, W. G. Graham, T. Morrow, and C. L. S. Lewis, Rev. Sci. Instrum. 70, 1801 (1999). 